Chemotherapy is generally used in treatment of systemic disease or in an attempt to prevent or delay systemic spread. The metastatic potential of MCTs varies and is dependent on several factors, such as grade of the tumour (based on Patnaik or Kiupel grading system), mitotic index (MI) and other factors of proliferation, clinical stage of disease, location of the MCT, clinical presentation and others.

Careful staging of a patient pre- or postsurgically is helpful in determining the extent of disease. Generally, MCTs tend to spread to locoregional lymph nodes, liver and spleen and less commonly to lungs.

Chemotherapy can be considered as primary treatment in non-resectable MCTs, in a neoadjuvant setting (presurgical in attempt to shrink the tumour), in patients with high-grade tumours, patients with confirmed spread or in cases with multiple MCTs; or as adjuvant treatment in patients with MCTs with increased risk of metastasis, or in cases of incompletely excised tumours.

Several systemic treatments and protocols have been proposed for patients diagnosed with MCTs with variable responses to treatment documented. The most commonly used protocol is the vinblastine/prednisolone protocol. This protocol is generally well tolerated and it has been shown to prolong survival in patients with highrisk MCTs (high grade, node-positive, mucous membrane origin). Tyrosine kinase inhibitors (TKIs) licensed for treatment of MCTs have become popular over recent years due to their simple administration, however careful consideration of when it would be appropriate to use these drugs is required, due to their potential adverse effects and the costs associated with treatment over time.

\section{KEY LEARNING OBJECTIVES}

- Differentiate between patients with high-risk and low-risk mast cel tumours (MCTs)
Identify a patient that would benefit from chemotherapy when diagnosed with MCT

- Gain an understanding of use of different chemotherapy protocols in patients with MCTs and ability to select the best protocol for a given patient

\section{MULTIPLE CHOICE QUESTIONS}

1. MCTs have a variable clinical and biological behaviour. Where do MCTs generally tend to spread to?
(A) Lymph node, liver and lungs
(B) Liver, spleen and lungs
(C) Lymph node, spleen and liver
(D) Lungs, lymph node and spleen

2. What is the most appropriate course of action for treatment of an incompletely resected stage-negative (no evidence of disseminated disease) low-grade MCT on a flank of a dog?
(A) A second more aggressive surgery
(B) Adjunctive chemotherapy
(C) Radiation followed by chemotherapy
(D) Radiation therapy

3. What is the mechanism of action of TKIs?

(A) TKIs inhibit spindle formation and cause cell death in $M$ phase of cell cycle

(B) TKIs are small molecules that target cell signalling

(C) TKIs are non-specific drugs that act on nucleus of the cell and cause apoptosis

(D) TKIs cause DNA cross-linkage that interferes with cell proliferation

\title{
Difficult mast cell tumours and dirty margins
}

\section{Nicholas Bacon \& Sarah L. Mason}

\section{WHY DO DIRTY MARGINS HAPPEN?}

Factors positively associated with achieving a clean surgical margin include:

- Definitive diagnosis prior to surgery

- Consideration of shrinking the mass ahead of surgery to consolidate peripheral margins

- Understanding the difference between metric margins (distance) and barrier margins (fascia) and how to apply these

- Surgical planning to include behavioural biology as a guide

- Taking into account that your desired reconstruction option should not compromise your margin of resection, i.e. have multiple plans.
Failure in any of these areas can result in residual disease, that is, a dirty margin.

\section{HOW CAN THEY BE PREVENTED?}

- Definitive diagnosis: minimally a fine-needle aspirate is indicated. Avoid incisional biopsies into mast cell tumours (MCTs) unless the definitive grade will significantly change your plan, this is rare

- Shrinking the mass ahead of surgery: use of prednisolone at $1 \mathrm{mg} / \mathrm{kg}$ for 7-14 days before surgery will reduce the diameter of most MCTs, we tend to reserve this when skin surrounding the tumour is at a premium, i.e. near the eye, planum, below the elbow and stifle, perineum

- Margins: there is no 'magic' margin for all MCTs. For many cutaneous MCTs, however, $2 \mathrm{~cm}$ skin margins and a fascial plane deep are sufficient. This may need to be increased in larger tumours, in the presence of inflammation, paraneoplastic signs, and if the deep fascia is of poor quality. In smaller quiescent tumours, 5-10 mm margins may be sufficient. In terms of incomplete margins, it is typically the deep margin that is insufficient 


\section{Oncology}

- Surgical planning: consider clinical behaviour, any cross-sectional imaging delineating gross tumour margins (pseudocapsule) and nodal and metastatic disease

- Multiple plans: be comfortable with a variety of reconstructive techniques, this will encourage the first resection to be appropriately sized, and so achieve a clean surgical margin first time.

\section{DO WE ALWAYS NEED TO INTERVENE AFTER INCOMPLETE MARGINS?}

Many incompletely excised MCTs will not recur, with recurrence rates reported in the region $22-38 \%$. For lowgrade tumours and some Patnaik intermediate grade tumours, active monitoring can be appropriate. Tumours with high risk features should be considered for additional locoregional therapy. This may well include removing the draining lymph node for more accurate staging. High-grade MCTs have a higher rate of recurrence and metastasis and additional therapy is recommended.

\section{WHAT CAN WE DO ABOUT INCOMPLETELY EXCISED MAST CELL TUMOURS?}

\section{Scar re-excision}

Depending on anatomical location, and the availability of local tissues, a single re-excision may hold some benefit to the patient.

\section{Adjunctive radiation}

Radiation therapy is commonly used in the microscopic disease setting, especially when a second resection is not possible or likely to achieve complete excision. The distal limb is a common location for this. The combination of planned marginal resection and adjunctive radiation can result in excellent outcomes with long-term local control rates over $93 \%$. Good quality presurgical photographs, accurate measurements and discussion with the radiation oncologist prior to surgery will maximize the chance for curative treatment.

\section{Systemic therapy: chemotherapy and targeted treatment}

Chemotherapy is commonly employed in the adjunctive treatment of canine MCT, the aim being to control or treat regional or systemic spread. Commonly utilized protocols include vinblastine/prednisolone and sometimes lomustine. Tyrosine kinase inhibitors, such as toceranib and masitinib, also have a role in the multimodal management of MCT.

\section{KEY LEARNING OBJECTIVES}

- Evaluate the surgical approach to canine mast cell tumours (MCTs)

- Appreciate the concept of presurgical planning of adjunctive therapy to improve local control of canine MCTs

- Develop understanding of when adjunctive treatment can improve outcomes in non-surgically cured MCTs

\section{MULTIPLE CHOICE QUESTIONS}

1. What is the likely recurrence rate for an incompletely excised Patnaik grade II canine cutaneous MCT without adjunctive treatment?
(A) Less than $20 \%$
(C) $40-60 \%$
(B) $20-40 \%$
(D) Greater than 60\%

2. Which of these MCT locations is most likely to be cured with scar excision of an incompletely excised tumour?
(A) Muzzle
(C) Perianal
(B) Lateral tarsal region
(D) Lateral thigh

3. Which adjunctive therapy has the best possibility of local control for a Patnaik grade II MCT of the distal antebrachium?
(A) High-dose radiotherapy (50-60 Gy)
(B) Moderate-dose radiotherapy (32-48 Gy)
(C) Re-excision
(D) Vinblastine chemotherapy

\section{Evaluating the canine lymphoma patient}

\section{Sarah L. Mason}

Lymphoma is a commonly presented neoplasia in dogs and while most commonly confined to the lymphoid tissues can affect any organ system. This lecture will discuss the approach to the canine lymphoma patient and treatment options, using case examples to illustrate common presentations and decision making in this condition.

The first steps in diagnosis are fine-needle aspiration (FNA) and cytology of affected lymph nodes, which is a fast and inexpensive route to diagnosis in many cases. Highgrade lymphoma is easily diagnosed by this method and in most cases the clinical pathologist will have confidence in making a diagnosis from good-quality cytological preparations. The prescapular or popliteal lymph nodes should be sampled when possible as the submandibular may present a more mixed picture, particularly in patients with dental disease as these nodes drain the oral cavity.

Occasional cases will require a biopsy, particularly when the cytology demonstrates populations of mixed or small lymphoid cells, as these are commonly lowgrade or indolent forms of the disease. In this situation lymphadenectomy is indicated to better classify the lymphoma and allow assessment of the architecture of the entire node. This avoids missing a focal lesion and helps in classifying disease. 